\title{
Bayesian Approach to Urinary ESBL-Producing Escherichia coli
}

José Miguel Sahuquillo-Arce ${ }^{1^{*}}$, Hèctor Perpiñán ${ }^{2}$, Carmen Armero $^{2}$, Antonio López-Quílez $^{2}$, María Selva $^{3}$ and Francisco González $^{3}$

${ }^{1}$ Grupo de Investigación de Infecciones Respiratorias, Hospital Universitari i Politècnic La Fe, Valencia, Spain

${ }^{2}$ Universitat de València, Valencia, Spain

${ }^{3}$ Dirección General de Salud Publica, CSISP, Valencia, Spain

*Corresponding author: José Miguel Sahuquillo-Arce, Grupo de Investigación de Infecciones Respiratorias, Hospital Universitari i Politècnic La Fe, Torre A, Bulevar Sur s/n, 46026 Valencia, Spain, Tel: 0034 963862744; Fax: 0034 961973177; E-mail: wadjur@hotmail.com

Received date: May 23, 2014 , Accepted date: July 02, 2014, Published date: July 09, 2014

Copyright: (C) 2014 Sahuquillo-Arce JM, et al. This is an open-access article distributed under the terms of the Creative Commons Attribution License, which permits unrestricted use, distribution, and reproduction in any medium, provided the original author and source are credited.

\begin{abstract}
This is a retrospective study about the prevalence of ESBL-producing Escherichia coli (EEC) in urinary specimens from patients from the Comunitat Valenciana from January 2007 to December 2008. Data were retrieved from RedMIVA, and Bayesian generalized linear mixed models were considered to study the prevalence of EEC with regard to demographical and microbiological factors.

The total number of infections considered was 164,502 , the amount of urinary isolates was 70,827 belonging to 49,304 different patients, and $5,161(7.3 \%)$ of the urinary isolates were EEC. Three out of four $E$. coli were isolated in women $(76.8 \%)$, men showed higher rates of EEC $(9.7 \%$ in men vs. $6.5 \%$ in women). EEC patients were, in average, 10.8 years older, and hospitalization was more frequent $(9.9 \%$ vs. $6.9 \%)$.

Resistance to non- $\beta$-lactams antimicrobials was higher in EEC. The rates of ciprofloxacin and co-trimoxazol resistance in EEC were $75.5 \%$ and $52.0 \%$, respectively, whereas it ranged between $1.4-12.4 \%$ for the rest of antimicrobials.

Prior EEC infection and hospitalization were the most relevant risk factors and increased the expected EEC probability approximately $400 \%$ and $50 \%$ respectively. Other infections played an important and positive role too, Enterobacteriaceae, P. aeruginosa and other bacteria being the most relevant elements. Female gender was a protective factor and reduced the risk by approximately $25 \%$ while age was an additive risk factor.

Finally, an open-access web-based software was constructed to compute the probability that an $E$. coli in a urinary infection be an EEC from a specific combination of risk factors. This pharmacovigilance tool should prove useful to monitor and control antimicrobial resistance spread.
\end{abstract}

Keywords: Pharmacovigilance; Control; Antimicrobial

\section{Introduction}

ESBL-producing Escherichia coli (EEC) has arisen and disseminated worldwide as an important cause of both nosocomial and community infections [1-4] and nowadays represents a major threat [5]. This has called for a re-evaluation of susceptibility interpretation and current antimicrobial therapies [6,7]. Early identification of potential EEC carriers is the first step to withhold the dispersal of these microorganisms and to avoid possible complications $[2,8,9]$. Therefore, surveillance for EEC at every level -i.e., national, regional, local or within the hospital- is critical in order to adequately design recommendations, for epidemiological and spread control purposes, as well as for an empirical treatment of infections caused by this microorganism, especially due to the complications derived from an inadequate antibiotherapy $[2,7,10]$.

The objective of this paper is to discuss the probability of carrying an EEC for a specific individual with a urinary tract infection (UTI), based on demographic and microbiological data easily retrieved from a medical record.

\section{Material and Methods}

\section{Study design}

This is a retrospective study about the prevalence of EEC in urinary specimens from patients from the Comunitat Valenciana, an autonomous region in the East of Spain with a population of 5,029,601 and a surface equal to $23,255 \mathrm{~km}^{2}$, which was divided geographically into 22 health departments at the time of the study.

Urinary isolates were obtained from January 2007 to December 2008. Microbial identification and antimicrobial susceptibility testing were performed according to each laboratory standard procedures and conforming to CLSI antimicrobial susceptibility criteria, either by regular biochemical reactions and agar diffusion susceptibility tests or by automated methods.

\section{Data collection}

Data were retrieved from the Comunitat Valenciana Microbiological Surveillance Network (RedMIVA), which daily compiles and analyzes information from 25 microbiology laboratories that manage more than $90 \%$ of the total population. Relevant 
demographic and microbiological data from all patients in the study were recorded. Such data included age, gender, health department, hospitalization by any cause and any other prior bacterial infection taking place during the study period. Likewise, information about susceptibility for the following antimicrobial agents was retrieved: amikacin, tobramycin, ciprofloxacin, fosfomycin, nitrofurantoin, and trimethoprim-sulfamethoxazole.

EEC was defined as an $E$. coli resistant to third generation cephalosporins but susceptible to amoxicillin-clavulanate. Thus most of the ESBLs encountered clinically -TEM, SHV and CTX-M-type enzymes (functional group 2be from the Bush-Jacoby-Medeiros classification) - were taken into account [11].

\section{Statistical methodology}

Bayesian generalized linear mixed models [12] were considered to study the prevalence of EEC with regard to the demographical and microbiological factors considered. Markov Chain Monte Carlo methods were applied for approximating the posterior distribution for the relevant parameters and hyperparameters of the model through the free software WinBUGS 1.4.3 [13].

We considered a general scenario with non-informative prior knowledge but, in order to avoid any chance of obtaining improper prior distributions, we have always worked with proper densities: Normal distributions with large variance $\left(10^{\wedge} 6\right)$ are elicited as the prior distribution for the coefficients of the model and, a hierarchical normal prior distribution for the geographical random effect. A vague uniform hyperprior distribution, Uniform $(0,2)$, for the standard deviation of that geographical effect is considered. In addition, we did a sensitivity analysis based on different Normal prior distributions for the regression coefficients with a wide range of possible values for the variance together with Uniform distributions for the standard deviation with values for the maximum larger than 2 . With all these elicitations we always obtained the same posterior distribution, and not only with the WinBUGS software but also through the R INLA package [14].

In order to construct the statistical model, the random variable Yij takes the value 1 when specimen i from a patient located in health department $\mathrm{j}\left(s_{\mathrm{ij}}\right)$ is ECC, and 0 otherwise. This variable was defined in terms of a Bernouilli distribution with probability $\mathrm{p}_{\mathrm{ij}}$, which is connected to the values of the different factors (gender, hospitalization, health department, presence or not of previous infections by $E$. coli, by EEC, by Enterobacter spp., by Enterobacteriaceae, by Pseudomonas aeruginosa, by Enterococcus faecalis, by Staphylococcus aureus and by Other bacteria) and covariate (age) in the study through the logit transformation:

$$
\begin{aligned}
& Y_{i j} \sim \operatorname{Bernoulli}\left(p_{i j}\right) \text {, } \\
& \operatorname{logit}\left(\mathrm{p}_{\mathrm{ij}}\right)=\alpha_{0}+\alpha_{1} \mathrm{l}_{\{\text {Women }}\left(\mathrm{S}_{i j}\right)+\alpha_{2} \mathrm{l}_{\{\text {Hospital }}\left(\mathrm{S}_{i j}\right)+ \\
& +\alpha_{3 \mathrm{k}} \mathrm{l}\left\{E_{\cdot} \cdot \operatorname{coli}\right\}\left(\mathrm{S}_{i j}\right)+\alpha_{3 \mathrm{k}} \mathrm{l}\left\{E_{E C C}\left(\mathrm{~S}_{i j}\right)+\alpha_{5} \mathrm{l}\left\{\text { Enterobacter }\left(\mathrm{S}_{i j}\right)\right.\right. \\
& +\alpha_{6} \mathrm{l}\{\text { Enterobacteriaceae }\}\left(S_{i j}\right)+\alpha 7\{\text { P. aeruginosa }\}\left(S_{i j}\right) \\
& +\alpha 8 \mathrm{l}\{\text { Other Bacteria }\}\left(\mathrm{S}_{i j}\right)+\alpha \mathrm{l}\{\text { E. faecalis }\}\left(\mathrm{S}_{i j}\right)+ \\
& +\alpha_{10} \mathrm{l}\{\text { S. a ureus }\}\left(\mathrm{S}_{i j}\right)+\alpha_{11} \text { Age }\left(\mathrm{S}_{i j}\right)+\mathrm{u}_{\mathrm{j}}
\end{aligned}
$$

where $\mathrm{I}_{\{\mathrm{A}\}}\left(s_{\mathrm{ij}}\right)$ stands for the indicator function defined as 1 if specimen $s_{\mathrm{ij}}$-i.e. the patient who has provided it- has had the characteristic $\mathrm{A}$, and zero otherwise (for example, $\mathrm{I}_{\{\mathrm{Woman}\}}\left(s_{\mathrm{ij}}\right)$ would be equal to 1 if the person providing specimen $s_{\mathrm{ij}}$ were a woman), Age $\left(s_{\mathrm{ij}}\right)$ is the age in years of the patient who supplies specimen $s_{\mathrm{ij}}$ and $\mathrm{u}_{\mathrm{i}}$ represents the random effect corresponding to health department $j$.

This model is re-parameterized considering as the baseline the group defined by men, non-hospitalized, with no previous infections by E. coli, by EEC, by Enterobacter spp., by Enterobacteriaceae, by $P$. aeruginosa, by $E$. faecalis, by $S$. aureus and by other bacteria. Note that the intercept $a 0$ in the model is the element that reflects the effect of this baseline group in the logit probability for ECC rather than directly on this probability.

\section{Results}

The total number of infections considered was 164,502, while the amount of urinary isolates analyzed was 70,827 belonging to 49,304 different patients. Of the urinary isolates, 5,161 (7.3\%) were categorized as EEC. Three out of four E. coli were isolated in women (76.8\%), however men showed higher rates of EEC (9.7\% in men vs. $6.5 \%$ in women). EEC patients were, in average, 10.8 years older, and hospitalization was more frequent too $(9.9 \%$ vs. $6.9 \%)$. Demographic data are summarized in Table 1.

\begin{tabular}{|l|l|l|l|}
\hline & E.coli & ESBL-E.coli & Total \\
\hline Number of isolates & 65,666 & 5,161 & 70,827 \\
\hline Mean Age (yrs) & 52.5 & 63.3 & 53.3 \\
\hline Gender & & & \\
\hline Women & $50,831(93.5)$ & $3,558(6.5)$ & 54,389 \\
\hline Men & $14,835(90.2)$ & $1,603(9.6)$ & 16,438 \\
\hline Hospitalization & & & \\
\hline Yes & $8,874(90.1)$ & $978(9.9)$ & 9,852 \\
\hline No & $56,792(93.1)$ & $4,183(6.9)$ & 60,975 \\
\hline
\end{tabular}

Table 1: Demographic data (n(\%)).

E. coli and EEC antimicrobial resistance data to different antimicrobial agents is displayed in table 2 . It is worth mentioning that the number of isolates fluctuates depending on the antimicrobial considered from 60,267 in the case of amikacin to 64,404 for ciprofloxacin. Resistance to non- $\beta$-lactams antimicrobials was considerably higher in EEC patients. In particular, the rates of ciprofloxacin and co-trimoxazol resistance were $75.5 \%$ and $52.0 \%$ respectively, whereas that rate ranges between $1.4 \%$ and $12.4 \%$ for the rest of antimicrobials.

Approximate posterior summaries of the statistical analysis are presented in table 3: posterior mean and 95\% credible interval for each of the unknown elements in the model and posterior mean and $95 \%$ probability interval for the odds ratio of each factor, except for the geographical one. The results are rather concluding since 0 is not included in the $95 \%$ credible interval of any risk factor. The sign of each posterior mean reveals if the corresponding risk factor acts increasing (+) or decreasing (-) the EEC probability.

A previous EEC infection appears as the most eminent risk factor and increases slightly more than four times the expected EEC probability. Hospitalization is the second most relevant factor and increases the expected EEC probability approximately $50 \%$. 
Page 3 of 5

\begin{tabular}{|l|l|l|l|}
\hline & E.coli & ESBL-E.coli & Total \\
\hline Amikacin & & & \\
\hline $\mathrm{n}$ & 60,267 & 5,022 & 65,289 \\
\hline Resistance & $173(0.3)$ & $68(1.4)$ & $241(0.4)$ \\
\hline Tobramycin & & & \\
\hline $\mathrm{n}$ & 62,775 & 5,058 & 67,833 \\
\hline Resistance & $3370(5.4)$ & $627(12.4)$ & $3997(5.9)$ \\
\hline Nitrofurantoin & & & \\
\hline $\mathrm{n}$ & 63,095 & 5,050 & 68,145 \\
\hline Resistance & $1018(1.6)$ & $197(3.9)$ & $1215(1.8)$ \\
\hline Ciprofloxacin & & & \\
\hline $\mathrm{n}$ & 64,404 & 5,127 & 69,531 \\
\hline Resistance & $17,889(27.8)$ & $3,870(75.5)$ & $21,759(31.3)$ \\
\hline Co-trimoxazol & & & \\
\hline $\mathrm{n}$ & 62,146 & 4,999 & 67,145 \\
\hline Resistance & $20,364(32.8)$ & $2,598(52.0)$ & $22,962(34.2)$ \\
\hline Fosfomycin & & & \\
\hline $\mathrm{n}$ & 62,789 & 4,999 & 67,788 \\
\hline Resistance & $1,337(2.1)$ & $219(4.4)$ & $1,556(2.3)$ \\
\hline
\end{tabular}

Other infections play an important and positive role in the EEC prevalence, Enterobacteriaceae, $P$. aeruginosa and other bacteria being the most relevant factors.

Age might seem rather insignificant, but since it is additive, it becomes relevant past middle age.

Female gender always acts as a protective factor; actually, being a woman reduces the risk by approximately $25 \%$ with regard to men.

\section{Discussion}

The major findings of this study are: i) the correlation between EEC and higher resistance levels to non- $\beta$-lactams antimicrobials, especially ciprofloxacin; ii) a higher proportion of EEC in men, older individuals, hospitalized patients and its association with previous infections, especially previous ECC; and iii) that the probability that an E. coli in a urinary infection be an EEC can be predicted for an individual with a specific combination of risk factors.

First of all, we would like emphasize that the aim of this paper was to approach EEC from a pragmatic point of view, since our goal was to build a statistical model to explain how different risk factors interact and modulate ECC acquisition in order to predict its emergence.

In agreement with other papers $[15,16]$, we have found a direct correlation between EEC and higher resistance levels to non- $\beta$-lactams antimicrobials commonly used for community or nosocomial UTI. It is well known that ESBL-carrying plasmids can also confer resistance to other antimicrobials [16-18], but the alarming level of resistance to ciprofloxacin in EEC among our population, which is higher than that of other studies conducted in Spain $[1,19,20]$, indicates a very close relationship in the transmission of ESBL and quinolone resistance which should be borne in mind by practitioners. Yet, amikacin, fosfomycin and nitrofurantoin remained as good therapeutic options due to the low resistance levels found, as described in prior studies [21]. All of this should be taken into account when issuing local guidelines for empirical antimicrobial treatment of UTI.

Risk factors inherent to the host like age or gender play an essential role in EEC occurrence. Older age is commonly accepted as a risk factor [4,22]; our study demonstrates how age is a constantly growing risk factor that becomes relevant from maturity on. This fact probably reflects a cumulative exposure to risk factors through time. Gender is a more controversial issue: our study agrees with Condoner et al. that found male gender as a risk factor, while female gender always acts as a protective factor, contrary to statements found in other papers [22-24].

Hospitalization is a commonly accepted risk factor [1]. In our study, it increased by $50 \%$ the probability of carrying an EEC. Possible explanations for this are higher antibiotic pressure, higher concentration of EEC and therefore, close contact and interaction with EEC carriers within the hospital setting. But, whatever the reason is, the item hospitalization acts as a surrogate of different risks factors [4], which leads us to the next point: previous infections.

Numerous studies have tried to identify which antimicrobials were more likely to increase EEC prevalence, but they often obtain different results. While many of them find third generation cephalosporins and quinolones as relevant key factors, others fail to obtain the same results; instead, they talk about second generation cephalosporins, penicillins or just "antibiotics" $[1,4,15,19,24-26]$. Furthermore, an antimicrobial that is found to be a risk factor in a bivariate analysis is not in a multivariate analysis in the same paper [23]. These 
discrepancies are most likely based on different antimicrobial policies, due to the prevalent ESBL enzymes or ESBL-carrying plasmids from each region [17], or even to the analysis methodology [27-29]. We tried to amend such potential confounders by identifying the infections and microorganisms involved that were relevant to our statistical modelling and use them as indicators of antimicrobial treatments. Eventually, we found out several microorganisms that were relevant. The first and more obvious factor was previous infections by EEC, indicating that the individual has been previously exposed to that microorganism and has apparently incorporated it into the bacterial flora. Next, infections by Enterobacteriaceae and $P$. aeruginosa; both of them are associated with the use of cephalosporins and quinolones, which are important for ECC emergence. $P$. aeruginosa is also related to patients with underlying conditions [30], and so are more likely associated to invasive procedures or antimicrobial prophylaxis. Other infections that were important in our model, all as independent risks factors, argue chiefly for the use of a wide range of antimicrobials. Interestingly, infections by Grampositive bacteria such as E. faecalis, intrinsically resistant to cephalosporins, seem also important and highlight the relevance of other antimicrobials in the selection and emergence of EEC.

Finally, all the data was used to construct a statistical model with the aims of explaining how different risk factors interact and describing EEC in our region. The most interesting feature of this model is its capacity for making predictions for EEC. For such reason, an open-access web-based implementation has been constructed in order to compute and display the posterior predictive distribution for an EEC regarding the values of the relevant risk factors and covariate from the last two years that could be accessible to every medical practitioner and, given the time, could be automated. This tool should be very useful for clinicians, microbiologists and epidemiologists when the time comes to prescribe empirical treatments for UTI or to prevent EEC from spreading. Moreover, our methodology can easily be used in other populations regardless of their antimicrobial policies or microorganism features.

One of the limitations of our study is that we retrieved the data from different hospitals and that these data were basically phenotypic. Studying the original bacteria in order to get genetic information would have been beyond price due to the large number of infections analyzed. Nevertheless, one of the major strengths of our study is precisely the great amount of data analyzed, using the same criteria for all the isolates.

In conclusion, we have been able to describe EEC in our population through Bayesian inference, providing our health system with a tool that could be essential for EEC control and surveillance. The use of a large database such as RedMIVA has been the cornerstone of this paper.

\section{Acknowledgments}

The authors would like to express their gratitude to all the microbiologist who collaborate with RedMIVA, without their effort, this paper would not be.

\section{Funding Details}

This research has been partially supported by the Ministerio de Ciencia e Innovación grant MTM2010-19528.

\section{References}

1. Calbo E, Romaní V, Xercavins M, Gómez L, Vidal CG, et al. (2006) Risk factors for community-onset urinary tract infections due to Escherichia coli harbouring extended-spectrum beta-lactamases. J Antimicrob Chemother 57: 780-783.

2. Oteo J, Pérez-Vázquez M, Campos J (2010) Extended-spectrum [beta]lactamase producing Escherichia coli: changing epidemiology and clinical impact. Curr Opin Infect Dis 23: 320-326.

3. Rodríguez-Baño J, Alcalá J, Cisneros JM, Grill F, Oliver A, et al. (2009) Escherichia coli producing SHV-type extended-spectrum beta-lactamase is a significant cause of community-acquired infection. J Antimicrob Chemother 63: 781-784.

4. Rodríguez-Baño J, Navarro MD (2008) Extended-spectrum betalactamases in ambulatory care: a clinical perspective. Clin Microbiol Infect 14 Suppl 1: 104-110.

5. Cantón R, Novais A, Valverde A, Machado E, Peixe L, et al. (2008) Prevalence and spread of extended-spectrum beta-lactamase-producing Enterobacteriaceae in Europe. Clin Microbiol Infect 14 Suppl 1: 144-153.

6. Cantón R (2010) [Interpretive reading of the antibiogram: a clinical necessity]. Enferm Infecc Microbiol Clin 28: 375-385.

7. Paterson DL, Bonomo RA (2005) Extended-spectrum beta-lactamases: a clinical update. Clin Microbiol Rev 18: 657-686.

8. Warren RE, Harvey G, Carr R, Ward D, Doroshenko A (2008) Control of infections due to extended-spectrum beta-lactamase-producing organisms in hospitals and the community. Clin Microbiol Infect 14 Suppl 1: 124-133.

9. Pitout JD, Laupland KB (2008) Extended-spectrum beta-lactamaseproducing Enterobacteriaceae: an emerging public-health concern. Lancet Infect Dis 8: 159-166.

10. Reynolds R (2009) Antimicrobial resistance in the UK and Ireland. J Antimicrob Chemother 64 Suppl 1: i19-23.

11. Livermore DM (2008) Defining an extended-spectrum beta-lactamase. Clin Microbiol Infect 14 Suppl 1: 3-10.

12. Christensen R, Johnson W, Branscum A, Hanson TE (2011) Bayesian Ideas and Data Analysis: An Introduction for Scientists and Statisticians. CRC Press, Taylor and Francis.

13. Lunn DJ, Thomas A, Best N, Spiegelhalter D (2000) WinBUGS - A Bayesian modelling framework: concepts, structure, and extensibility. Statistics and Computing 10: 325-337.

14. Rue H, Martino S, Chopin N (2009) Approximate Bayesian inference for latent Gaussian models using integrated nested Laplace approximations (with discussion). Journal of the Royal Statistical Society B 71: 319-392.

15. Azap OK, Arslan H, SerefhanoÄŸlu K, ColakoÄŸlu S, ErdoÄŸan H, et al. (2010) Risk factors for extended-spectrum beta-lactamase positivity in uropathogenic Escherichia coli isolated from community-acquired urinary tract infections. Clin Microbiol Infect 16: 147-151.

16. Falagas ME, Karageorgopoulos DE (2009) Extended-spectrum betalactamase-producing organisms. J Hosp Infect 73: 345-354.

17. Coque TM, Baquero F, Canton R (2008) Increasing prevalence of ESBLproducing Enterobacteriaceae in Europe. Euro Surveill 13.

18. Poirel L, Pitout JD, Calvo L, Rodriguez-Martinez JM, Church D, et al. (2006) In vivo selection of fluoroquinolone-resistant Escherichia coli isolates expressing plasmid-mediated quinolone resistance and expanded-spectrum beta-lactamase. Antimicrob Agents Chemother 50: 1525-1527.

19. Rodríguez-Baño J, Picón E, Gijón P, Hernández JR, Ruíz M, et al. (2010) Community-onset bacteremia due to extended-spectrum beta-lactamaseproducing Escherichia coli: risk factors and prognosis. Clin Infect Dis 50: 40-48.

20. Baquero F, Cercenado E, Cisterna R, de la Rosa M, García-Rodríguez JA, et al. (2006) [Patterns of susceptibility to antibiotics of Enterobacteriaceae causing intra-abdominal infection in Spain: SMART 2003 study outcomes]. Rev Esp Quimioter 19: 51-59. 
Citation: Sahuquillo-Arce JM, Perpiñán H, Armero C, López-Quílez A, Selva M, et al. (2014) Bayesian Approach to Urinary ESBL-Producing Escherichia coli . J Pharmacovigilance 2: 133. doi:10.4172/2329-6887.1000133

Page 5 of 5

21. Sahuquillo-Arce JM, Selva M, Perpiñán H, Gobernado M, Armero C, et al. (2011) Antimicrobial resistance in more than 100,000 Escherichia coli isolates according to culture site and patient age, gender, and location. Antimicrob Agents Chemother 55: 1222-1228.

22. Pitout JD, Hanson ND, Church DL, Laupland KB (2004) Populationbased laboratory surveillance for Escherichia coli-producing extendedspectrum beta-lactamases: importance of community isolates with blaCTX-M genes. Clin Infect Dis 38: 1736-1741.

23. Topaloglu R, Er I, Dogan BG, Bilginer Y, Ozaltin F, et al. (2010) Risk factors in community-acquired urinary tract infections caused by ESBLproducing bacteria in children. Pediatr Nephrol 25: 919-925.

24. Peña C, Gudiol C, Tubau F, Saballs M, Pujol M, et al. (2006) Risk-factors for acquisition of extended-spectrum beta-lactamase-producing Escherichia coli among hospitalised patients. Clin Microbiol Infect 12: 279-284.

25. Colodner R, Rock W, Chazan B, Keller N, Guy N, et al. (2004) Risk factors for the development of extended-spectrum beta-lactamaseproducing bacteria in nonhospitalized patients. Eur J Clin Microbiol Infect Dis 23: 163-167.
26. Ena J, Arjona F, Martínez-Peinado C, López-Perezagua Mdel M, Amador C (2006) Epidemiology of urinary tract infections caused by extendedspectrum beta-lactamase-producing Escherichia coli. Urology 68: 1169-1174.

27. Hyle EP, Bilker WB, Gasink LB, Lautenbach E (2007) Impact of different methods for describing the extent of prior antibiotic exposure on the association between antibiotic use and antibiotic-resistant infection. Infect Control Hosp Epidemiol 286: 647-654.

28. Hyle EP, Gasink LB, Linkin DR, Bilker WB, Lautenbach E (2007) Use of different thresholds of prior antimicrobial use in defining exposure: impact on the association between antimicrobial use and antimicrobial resistance. J Infect 55: 414-418.

29. MacAdam H, Zaoutis TE, Gasink LB, Bilker WB, Lautenbach E (2006) Investigating the association between antibiotic use and antibiotic resistance: impact of different methods of categorising prior antibiotic use. Int J Antimicrob Agents 28: 325-332.

30. Rossolini GM, Mantengoli E (2005) Treatment and control of severe infections caused by multiresistant Pseudomonas aeruginosa. Clin Microbiol Infect 11: 17-32. 